\title{
Comparative study of electronic-structure methods for platinum-containing molecules: bond lengths and bond dissociation energies ${ }^{\star \star \star}$
}

\author{
Daniel Süß, Stefan E. Huber ${ }^{\mathrm{a}}$, and Andreas Mauracher ${ }^{\mathrm{b}}$ \\ Institute of Ion Physics and Applied Physics, University of Innsbruck, Technikerstraße 25, 6020 \\ Innsbruck, Austria
}

Received 19 December 2018 / Received in final form 3 April 2019

Published online 2 July 2019

(C) The Author(s) 2019. This article is published with open access at Springerlink.com

\begin{abstract}
We compare various electronic structure methods including a broad range of density functionals with experimental data on bond lengths and bond dissociation energies available for di- and triatomic platinum-containing molecules. In total we employ 54 GGA, 17 meta-GGA, 36 hybrid, 103 mixed GGA/meta GGA, 17 hybrid, 7 meta hybrid, 10 range-separated hybrid and 5 double hybrid density functionals. Furthermore, the performance of ab initio methods including Hartree-Fock, Møller-Plesset perturbation theory up to fourth order as well as coupled cluster theory up to perturbatively approximated triple excitations, i.e. $\operatorname{CCSD}(\mathrm{T})$, is also investigated. In the case of bond lengths, the smallest mean average deviation from experimental values yielding $0.3 \mathrm{pm}$ is found for the hybrid density functional TPSSh. Interestingly, neither recent double hybrid functionals nor ab initio methods result in similar, commensurable accuracies. For the investigated bond dissociation energies, the GGA functional TPSSVWN5 is the closest to experiment with deviations of $6.97 \mathrm{kcal} / \mathrm{mol}$. Finally, we address various possible sources of errors that may explain the large mean average deviation from experiment in the case of $\operatorname{CCSD}(\mathrm{T})(8.87 \mathrm{kcal} / \mathrm{mol})$, including the effect of basis set size, the influence of the multireference character of the molecular wave function, the quality of the HF determinant as reference wave function and the influence of core electron correlation.
\end{abstract}

\section{Introduction}

Platinum containing molecules are up to now among the leading drugs used in anticancer chemotherapy and cover substances such as cisplatin (cis-diamminedichloridoplati num(II)), carboplatin (cis-diammine(cyclobutane-1,1-dica rboxylate-O, $\left.\mathrm{O}^{\prime}\right)$ platinum(II)), oxaliplatin $([(1 R, 2 R)$-cl ocyhexane-1,2-diamine] (ethanedioato-O,O') platinum(II)) and many more [1]. Despite the clinical usefulness of those so far developed and approved cytostatic agents, they still exhibit major drawbacks which restrict their usage. Dose-limiting side effects include nephrotoxicity, ototoxicity and neurotoxicity, high reactivity and limited solubility, intrinsic and/or acquired resistances and the uncomfortable and cost intensive way of administration via infusion [2]. Unsurprisingly, much research has been devoted to overcome these limitations [3,4]. Even a restricted scan of only platinum-containing molecules

* Contribution to the Topical Issue "Dynamics of Systems on the Nanoscale (2018)", edited by Ilko Bald, Ilia A. Solov'yov, Nigel J. Mason and Andrey V. Solov'yov.

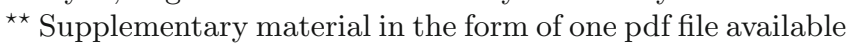
from the Journal web page at https://doi.org/10.1140/epjd/e2019-90691-1

a e-mail: s.huber@uibk.ac . at

b e-mail: andreas.mauracher@uibk.ac.at has still to cover a vast variety of possible candidate molecules. Therefore, providing as good as possible theoretical and/or empirical guidance for a systematic design of considered drugs appears essential. In particular, in order to design metal-organic drugs with improved pharmacological profiles, details of the modes of action, toxicity and resistance need to be studied, understood and linked to underlying molecular properties. Thus, relations may be discovered which reveal how properties at the microscopic, i.e. molecular, level and the macroscopic one, i.e. the efficacy of a considered drug in clinical research, are connected. Such approaches are well-established in pharmaceutical research, e.g. in form of (quantitative) structure-activity relationship (QSAR or SAR) and related theoretical studies which have been applied also in the present context $[2,5,6]$.

However, the outcome of such studies depends heavily on the reliability and validity of the numerical, typically quantum chemical, methods employed to compute properties at the molecular level. In general, computational chemistry offers a broad variety of methods which vary substantially in both achievable accuracy as well as computational cost depending on the size of the chemical system under consideration. For system sizes typical for the research framework described above, a viable balance between reasonable accuracy and manageable computational cost is delivered 
by density functional theory (DFT). The validation of DFT for a certain application requires comparison of representative molecular properties to reliable experimental or higherlevel theoretical data or both. For main group chemistry, coupled cluster (CC) theory provides such higher-level theoretical methods which are able to deliver benchmarkquality data [7]. Especially CC including single and double excitations with a quasi-perturbative treatment of connected triple excitations, i.e. $\operatorname{CCSD}(\mathrm{T})[8,9]$, became known as a "gold standard" in computational chemistry due to its often delivered high accuracy. In the light of the scarcity of reliable experimental molecular properties for systems containing transition metals like platinum, it therefore appears appropriate to use reference data derived by $\operatorname{CCSD}(\mathrm{T})$ in order to validate computationally less demanding DFT approaches. Unfortunately, the situation is more difficult and controversial in this case than for main group chemistry. Whereas Truhlar and co-workers [10] showed that $\operatorname{CCSD}(\mathrm{T})$ does not generally deliver benchmark quality data for systems containing transition metals, Dixon and co-workers [11] could scrutinize the importance of the inclusion of core electrons and extrapolation to the complete basis set (CBS) limit in order to achieve this goal.

In this work, we revise some of these findings by comparison of results obtained with diverse electronic-structure methods with available experimental gas-phase data on molecular geometries and energies, however focusing specifically on platinum-containing molecules. In particular, we compare the results of various ab initio approaches (from Hartree-Fock (HF) theory up to $\operatorname{CCSD}(\mathrm{T})$ ) as well as DFT methods (including GGA, meta GGA, hybrid, meta hybrid, range-separated hybrid and double hybrid functionals) with experimental data for ten bond lengths and ten bond dissociation energies (BDEs) of di- and triatomic platinumcontaining molecules. Although admittedly small, the size of our test sets for these molecular properties reflects the scarcity of available experimental data. To the best of our knowledge, no such gas-phase data for the larger anticancer compounds have been reported so far. Note that is seems not advisable in this context to use molecular structures and bond energies derived from corresponding solid state structures. It has been shown by Georgieva et al. [12] that the intermolecular hydrogen bonding network of crystalline cisplatin significantly influences the structural pattern of cisplatin.

Moreover, we compare our findings with conclusions drawn from earlier studies employing $\operatorname{CCSD}(\mathrm{T})$ for the calculation of reference data in order to validate and assess the performance of a variety of DFT methods for calculation of geometric and energetic properties of platinum-containing anticancer compounds [13]. To this end, we critically discuss the importance of a careful validation of high-level theory results when confronted with transition metal chemistry.

\section{Methods}

\subsection{Test sets}

To assess the validity and reliability of the various quantum chemical methods compared in this work,
Table 1. Test sets for assessing validity and reliability concerning bond lengths and BDEs for platinum containing molecules considered in this work (references for the experimental data are given in squared brackets).

\begin{tabular}{lclc}
\hline & TS-BL & \multicolumn{2}{c}{ TS-BDE } \\
\hline Molecule & Bond length $(\AA)$ & Molecule & BDE $(\mathrm{eV})$ \\
\hline $\mathrm{PtH}$ & $1.528[14]$ & $\mathrm{PtC}^{+}$ & $5.95[15]$ \\
$\mathrm{PtC}$ & $1.678[16]$ & $\mathrm{PtC}^{+}$ & $5.46[17]$ \\
$\mathrm{PtN}$ & $1.682[16]$ & $\mathrm{PtN}^{+}$ & $3.84[18]$ \\
$\mathrm{PtO}$ & $1.73[16]$ & $\mathrm{PtN}^{+}$ & $3.35[18]$ \\
$\mathrm{PtF}$ & $1.8743[16]$ & $\mathrm{PtO}^{+}$ & $4.30[15]$ \\
$\mathrm{PtSi}$ & $2.0615[16]$ & $\mathrm{PtO}^{+}$ & $3.26[18]$ \\
$\mathrm{PtS}$ & $2.0398[16]$ & $\mathrm{PtCO}^{+}$ & $2.28[17]$ \\
$\mathrm{PtCl}$ & $2.1527[16]$ & $\mathrm{PtNO}^{+}$ & $3.13[18]$ \\
$\mathrm{PtCN}$ & $1.8987[18]$ & $\mathrm{OPtO}^{+}$ & $4.41[15]$ \\
$\mathrm{PtCO}$ & $1.7604[18]$ & $\mathrm{OPtO}^{+}$ & $3.06[15]$ \\
\hline
\end{tabular}

experimental bond lengths and BDEs for platinumcontaining molecules were compiled (Tab. 1). The test set for assessing validity and reliability concerning the considered bond lengths consists of eight diatomic and two triatomic neutral molecules and is denoted as TS-BL for the remainder of this work. The test set for assessing validity and reliability concerning BDEs consists of ten molecules in total, again including diatomic and triatomic molecules as well as neutral and positively charged species and is denoted as TS-BDE for the remainder of this work. The errors given in the experimental works concerning BDEs are always smaller than $0.15 \mathrm{eV}$. Note that concerning the triatomic compounds in both test sets the given experimental quantity refers to the bond involving the platinum atom, e.g. in the case of $\mathrm{PtCN}$ it refers to the $\mathrm{Pt}-\mathrm{C}$ bond in the molecule. All triatomic molecules considered here yield (nearly) linear ground state geometries in their ground states. Please note, that experimental bond lengths contain zero-point vibrational contributions. The bond lengths are obtained from accurate measurements of effective rotational constants, which omit higher order effects, for details see e.g. reference [16].

\subsection{Quantum chemical calculations}

In order to cover a broad variety of electronic structure methods we considered the following quantum chemical approaches. Concerning ab initio methods we included HF theory, Møller-Plesset perturbation theory up to fourth order (MP2 [19-23], MP3 [24,25], MP4 $[25-27])$ as well as coupled cluster theory up to perturbatively approximated triple excitations (CCD [28], CCSD [29-31], $\operatorname{CCSD}(\mathrm{T})[8,32])$. Concerning DFT we included 54 GGA, 17 meta-GGA, 36 hybrid, 103 mixed GGA/meta GGA, 17 hybrid, 7 meta hybrid, 10 rangeseparated hybrid and 5 double hybrid density functionals. A complete list of all employed functionals and further computational details are given in Supplementary material (SI).

To allow a proper comparison of our findings with those of the earlier work by Kokoschka et al. [13], see Section 1, 
the def2-QZVP basis set of Weigend and Ahlrichs [33] was used in all calculations. Note further that Kokoschka et al. [13] based their study on crystallographic data and hence used the COSMO solvation model to simulate the crystal environment, whereas we refer to experimental gas-phase data.

The def2-QZVP basis set applies an effective core potential (ECP) for platinum. Both the restricted basis set size as well as the neglect of explicit treatment of core electrons serve as possible sources of inaccuracy. The possible implications are discussed in Section 3.3 in addition to the effects of multireference character and suitability of the $\mathrm{HF}$ determinant as reference wave function for coupled cluster theory [34].

In a first step, the geometries of all molecules were optimized. BDEs were calculated by

$$
\mathrm{BDE}(\mathrm{AB})=\mathrm{E}(\mathrm{AB})-\mathrm{E}(\mathrm{A})-\mathrm{E}(\mathrm{B})
$$

where $\mathrm{BDE}(\mathrm{AB})$ denotes the bond dissociation energy of the molecule $\mathrm{AB}, \mathrm{E}(\mathrm{AB})$ is the electronic energy of the molecule $\mathrm{AB}$ at the optimized geometry, $\mathrm{E}(\mathrm{A})$ and $\mathrm{E}(\mathrm{B})$ are the electronic energies of the (optimized) fragments A and B, respectively. We included corrections for zero-point energy and basis set superposition error in all calculations.

Finally, we computed the mean average deviation (MAD) from experimental values as a measure for accuracy and the standard deviation (SD) as a measure for precision for all considered methods as given below. The MAD is given by

$$
\mathrm{MAD}=\frac{1}{N} \sum_{i}^{N}\left|x_{i}-V_{i}\right|,
$$

where $N$ is the number molecules in the respective test set (10 for each of TS-BL and TS-BDE), and $x_{i}$ and $V_{i}$ are the calculated and experimental values of the $i$ th molecule in the test set, respectively.

The SD is given by

$$
\mathrm{SD}=\sqrt{\frac{\sum_{i=1}^{N}\left(a_{i}-\bar{a}\right)^{2}}{N}},
$$

where $a_{i}=x_{i}-V_{i}$, and $\bar{a}=\frac{1}{N} \sum_{i}^{N} a_{i}$ denotes the mean value of all $a_{i}$.

\section{Results and discussion}

Our results are organized as follows. First, we analyze the performance of various electronic structure methods including many density functionals and (post)-HF methods concerning the reproduction of experimentally accurately measured bond lengths contained in our test set TS-BL, see Table 1. This is followed by a discussion on the reliability of calculated BDEs considering the molecules in our test set TS-BDE, see also Table 1. Finally, we address possible causes which may underlie the resulting, comparably large deviation from experimental BDEs in the case of $\operatorname{CCSD}(\mathrm{T})$.

\subsection{Bond lengths}

The specific bond lengths of ten molecules containing platinum considered in TS-BL, see Table 1, were derived at various levels of theory by optimizing the molecular geometries. A full compilation of all optimized bond lengths of all compared methods is given in Supplementary material. To analyze the obtained data, we calculate the MAD from experimental data and the SD for the calculated bond lengths. In Figure 1, we present a selection of our results. All tested ab initio methods are shown, whereas concerning density functionals, we show only the three most accurate and precise of each considered class. The bars representing the MADs and SDs of ab initio methods are coloured in red, and the bars representing the MADs and SDs of density functionals belonging to the group of GGA, meta GGA, mixed GGA, hybrid, meta hybrid, range separated hybrid and double hybrid functionals, are coloured in blue, yellow, green, violet, grey, light red and light blue, respectively.

Considering these eight groups shown in Figure 1, it is found that ab initio methods and double hybrid functionals yield higher MADs than meta GGA functionals and hybrid functionals for the considered molecules. In particular, from all tested methods, the range-separated meta hybrid density functional TPSSh [35] yields the smallest MAD of $0.3 \mathrm{pm}$ followed by the meta hybrid functional tHCTHhyb [36] and the range-separated hybrid functional HSE03 [37-42] yielding MADs of 0.45 and 0.57 pm, respectively. All three functionals yield thus smaller MADs than CCSD(T) which yields a MAD of $0.62 \mathrm{pm}$ and will be discussed in more detail below. Hence, the inclusion of a mixture of HF exchange with DFT exchange-correlation in the case of different types of hybrid functionals appears especially important in order to yield the most accurate bond lengths with a reasonable increase in the computational costs compared to GGA functionals which are discussed further below. Another, widely used hybrid density functional, in particular in organic chemistry, is B3LYP [43]. For B3LYP it is known, that it achieves reasonable results for a wide range of organic molecules. However, in the case of our test set, B3LYP yields a MAD of $0.88 \mathrm{pm}$, which is 2.5 times higher than the MAD of TPSSh at comparable computational costs.

The relative deviations of the most accurate of the tested (meta-)GGA density functionals are very close to each other, yielding about $0.80 \mathrm{pm}$, see also Figure 1 . In particular, the functionals TPSSPBE (mixed meta GGA and pure GGA), TPSSPW91 (mixed meta GGA and pure GGA), TPSSTPSS (meta GGA) and TPSSVP86 (mixed meta GGA and pure GGA) yield MADs of $0.79,0.80$, 0.80 and $0.82 \mathrm{pm}$, respectively. Note that we use here the convention to specify the pure functional by combining the exchange functional component with the one for the correlation functional; hence, TPSSPBE denotes using the (meta GGA) TPSS [44] exchange functional and the (pure GGA) PBE [45] correlation functional.

It is noteworthy, that all of these functionals have the TPSS exchange part in common which is also included in the hybrid functional TPSSh yielding the smallest 

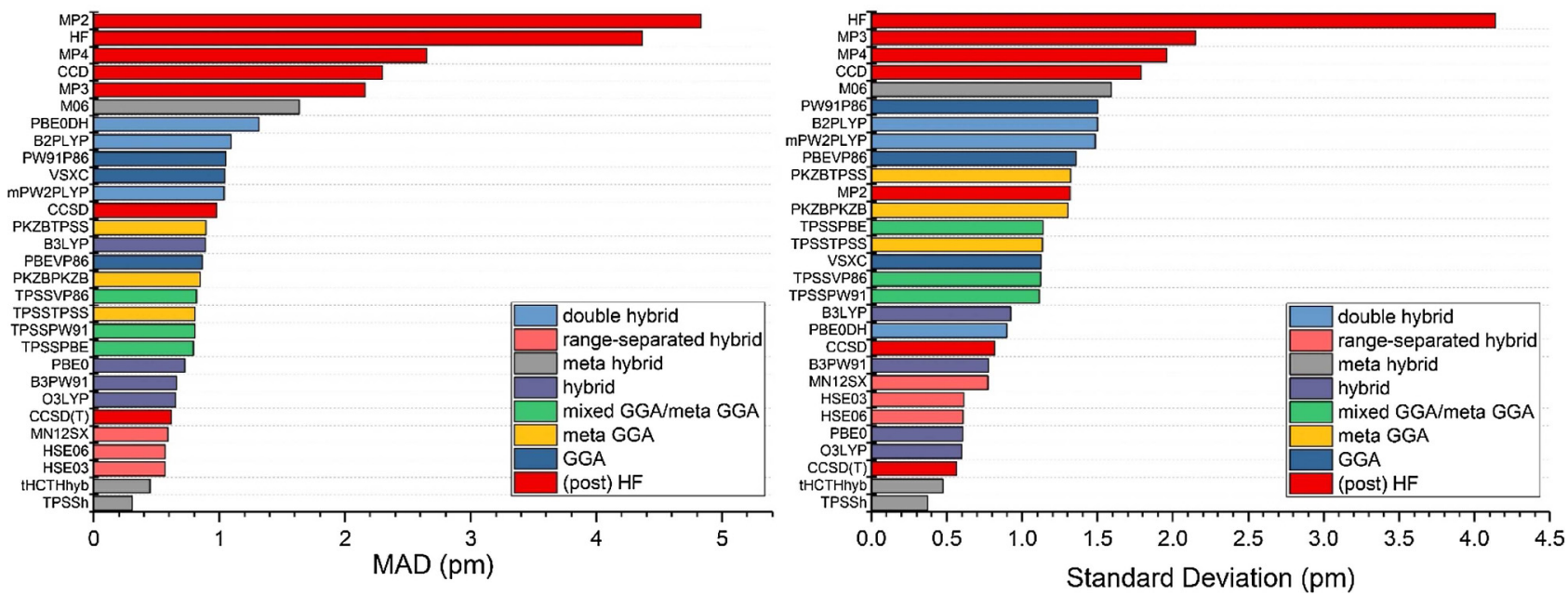

Fig. 1. MADs (left) and SDs (right) for bond lengths obtained with various computational methods in conjunction with the def2-QZVP basis set.

overall MAD as discussed above. Apart from PBE the other correlation functionals are PW91 [44,46,47], TPSS [44] and VP86 $[48,49]$. The (meta) GGA functionals might be kept in mind to obtain still reliable geometrical information of larger systems, for which hybrid functionals become computationally too expensive. This might become relevant especially for large-scale computations including geometrical (pre-)screening procedures.

Interestingly, Figure 1 also shows a comparably rather large deviation of all considered double hybrid density functionals. For instance, Grimme's B2PLYP [50] functional yields a MAD of $1.09 \mathrm{pm}$, which is 3.5 times higher than the MAD obtained with TPSSh at significantly higher computational cost due to the inclusion of perturbatively-calculated corrections.

(Post)-HF methods yield MADs above $2 \mathrm{pm}$ except for $\operatorname{CCSD}$ and $\operatorname{CCSD}(\mathrm{T})$. The highest MAD of this class of methods is obtained with MP2, which yields a MAD of $4.83 \mathrm{pm}$. All bond lengths optimized with MP2 are shorter than their experimental reference value. This is in agreement with the known tendency of MP2 to overbind in the case of transition metals [51]. This is also observed for $\operatorname{CCSD}(\mathrm{T})$, albeit with a considerably smaller MAD $(0.62$ $\mathrm{pm})$. In an earlier work [13] it was concluded, that hybrid functionals tend to yield too long bond lengths. However, it should be noted that the authors in said publication used the optimized geometry derived from B2PLYP as reference value for the comparison with other density functionals. This choice was based on the small deviation of B2PLYP bond lengths in comparison to one dimensional potential energy surface scans at the $\operatorname{CCSD}(\mathrm{T})$ level of theory. However, in the case of the molecules considered by us B2PLYP yields (except for PtS and PtSi) a similar trend of overbinding as does $\operatorname{CCSD}(\mathrm{T})$.

Besides knowing how far a method's results are from reliable experimental values on average, it is also important how much the accuracy of a method varies with the considered systems. This is explored by us using the SD of the deviations from experimental values of the considered methods, see Section 2.2. We note that the result- ing MADs and SDs are correlated, i.e. methods yielding a small MAD mostly also yield a small SD. Hence, the mentioned methods do not only yield the most accurate results, but are also the most precise methods. An exceptional case concerning the noted correlation represents MP2, which yields relatively mediocre but interestingly better precision than both MP3 and MP4, but by far the largest MAD of all tested methods. The reason for that is the above mentioned systematic overbinding of MP2 in the case of molecules containing transition metals.

\subsection{Bond dissociation energies}

We compared ten different, experimentally determined bond dissociation energies of small platinum-containing molecules (see Tab. 1). A selection of our results is depicted in Figure 2 using the same categorization scheme concerning shown methods as in our discussion of bond lengths above. Please note that we included corrections for zero-point energy and basis set superposition error in all calculations.

We find that the most accurate methods are comprised solely of GGA and hybrid functionals, see Figure 2. The most accurate functional, i.e. the one with the smallest MAD, is the combination of meta GGA correlation and LDA exchange functionals TPSSVWN5 [44,48] followed by the hybrid functionals B971 [52] and B98 [53] yielding $6.97,7.41$ and $7.47 \mathrm{kcal} / \mathrm{mol}$, respectively. Both double hybrid functionals as well as ab initio methods yield considerably larger MADs, see Figure 2. For instance, B2PLYP yields a MAD of $10.16 \mathrm{kcal} / \mathrm{mol}$. For comparison, the most accurate double hybrid functional mPW2PLYP yields a MAD of $8.78 \mathrm{kcal} / \mathrm{mol}$. These MADs represent a remarkably large deviation in light of the accuracy of these functionals found for molecules consisting of main group elements. For instance, for the molecules compiled in the G2/97 test set a deviation of below $2 \mathrm{kcal} / \mathrm{mol}$ has been reported [50]. However, B2PLYP's large MAD is mainly due to the deviation for the molecular ion 

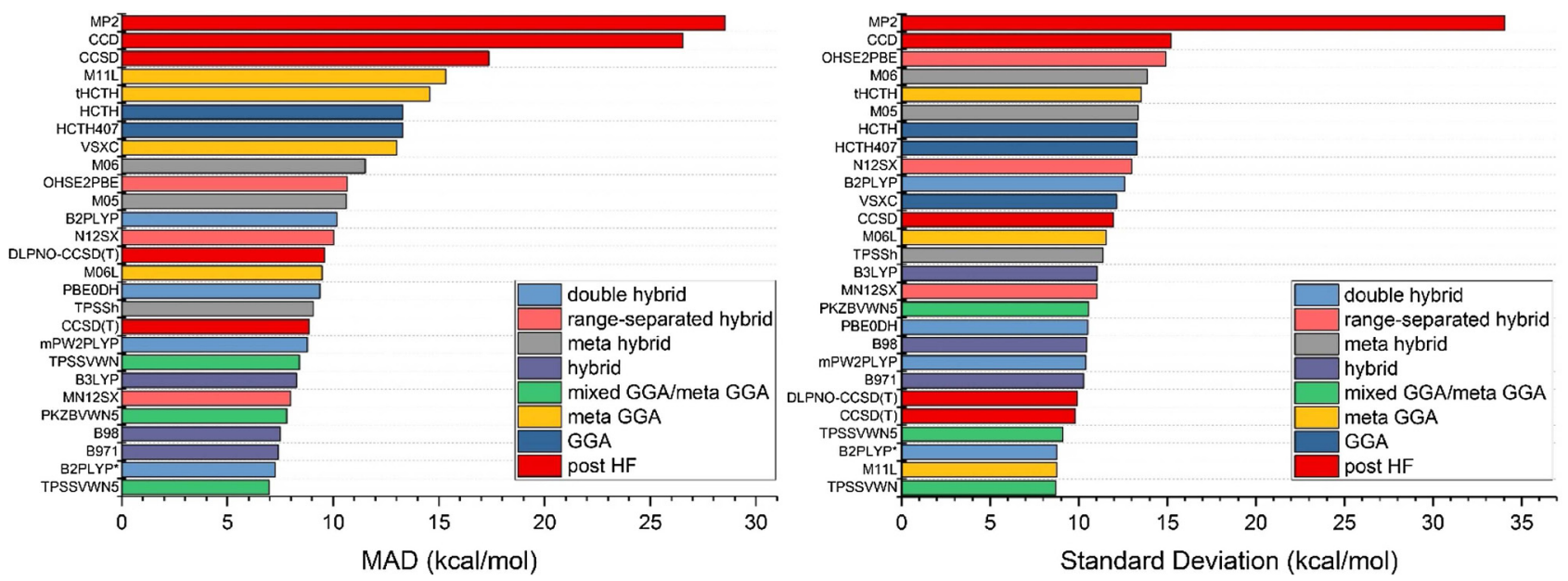

Fig. 2. MADs (left) and SDs (right) for bond dissociation energies obtained with various computational methods in conjunction with the def2-QZVP basis set. The notation B2PLYP* indicates the MAD and SD resulting for B2PLYP when the molecular ion $\mathrm{OPtO}^{+}$is omitted from the test set TS-BDE.

$\mathrm{OPtO}^{+}(36.2 \mathrm{kcal} / \mathrm{mol})$. If this outlier is omitted, the corresponding MAD of B2PLYP becomes $7.26 \mathrm{kcal} / \mathrm{mol}$, yielding then the second smallest MAD. This result is indicated by the notation B2PLYP* in Figure 2.

The MADs of the tested ab initio methods range from $8.87 \mathrm{kcal} / \mathrm{mol}$ for CCSD(T) and $125 \mathrm{kcal} / \mathrm{mol}$ for HF. Like in the case of bond lengths, also for the considered BDEs, MP2 yields a remarkably large MAD of $28.5 \mathrm{kcal} / \mathrm{mol}$. This may be related - to some extent - also to the poor performance of double hybrid functionals, which combine contributions from exact $\mathrm{HF}$ exchange and an MP2-like correlation to pure DFT exchange and correlation. Moreover, the deviation of MP2 results in BDEs which are consistently higher than the corresponding experimental values for all considered molecules. This finding is in line with the overbinding found for MP2 discussed already in Section 3.1. Also in the case of $\operatorname{CCSD}(\mathrm{T})$ we find a remarkably large MAD of $8.87 \mathrm{kcal} / \mathrm{mol}$, which is significantly larger than for many other systems [7], especially not containing transition metals. We note further that the computationally cheaper DLPNO-CCSD(T) method [54] yields with $9.58 \mathrm{kcal} / \mathrm{mol}$ only a slightly larger MAD than CCSD(T). Possible causes which may underlie the resulting, comparably large deviation from experimental data in the case of CCSD $(\mathrm{T})$ are discussed in detail in Section 3.3.

Concerning the precision of the assessed methods, we note that the correlation between accuracy and precision is considerably weaker in the case of the assessed BDEs than it was for the assessed bond lengths. For instance, the most accurate functional TPSSVWN5 appears at rank four concerning precision. Vice versa, the most precise functional TPSSVWN appears at rank eight concerning its accuracy.

Given that both accuracy and precision are usually desired at the same time, these findings interestingly support the use of some GGA functionals instead of both computationally considerably more expensive and methodologically more involved hybrid and double hybrid functionals as well as ab initio methods. Together with our findings concerning the assessed bond lengths in
Section 3.1, our results are quite in contrast to the usual textbook procedure (at least for large-scale computations) which typically suggests the opposite, i.e. to use a computationally "cheap" GGA functional for optimization purposes and "refine" the computation of the energy of the system by a more elaborate method.

\subsection{Discussion of error sources in the $\operatorname{CCSD}(T)$ calculations}

$\operatorname{CCSD}(\mathrm{T})$ calculations in conjunction with basis sets of similar size as def2-QZVP yield MADs of about $1 \mathrm{kcal} / \mathrm{mol}$ for test sets containing atoms and molecules of the first and second rows $[7,55]$. For test sets including transition metals, the MAD can be substantially larger [34] as we observed here in the case of platinum-containing molecules resulting in an overall MAD of $8.87 \mathrm{kcal} / \mathrm{mol}$. Nevertheless, in the following we discuss which factors might contribute to such a high MAD and we aim to quantify the possible contributions. The discussed contributions are the multireference character of the molecular wave function, the basis set size, the use of a Hartree-Fock wave function as reference wave function and the treatment of core electrons.

Basis set size. To estimate the error stemming from a limited basis set size, we calculated the bond dissociation energies also with smaller basis sets and extrapolated the results also to the complete basis set limit (CBS-limit) as implemented in the software ORCA [56]. In brief, given two basis sets of the same class (like Dunning's correlation consistent or Ahlrich's def2 basis sets) with cardinal number $X$ and $Y$ with $X>Y$, extrapolation of the total energy to the CBS-limit can be achieved via the formula

$$
E_{\mathrm{SCF}}^{(X)}=E_{\mathrm{SCF}}^{(\infty)}+A \cdot \exp (-\alpha \sqrt{X}),
$$

where $E_{\mathrm{SCF}}^{(\infty)}$ is the SCF energy in the CBS-limit, $E_{\mathrm{SCF}}^{(X)}$ is the SCF energy calculated with the basis set with cardinal number $X$, and $A$ and $\alpha$ are constants. Equation (4) 


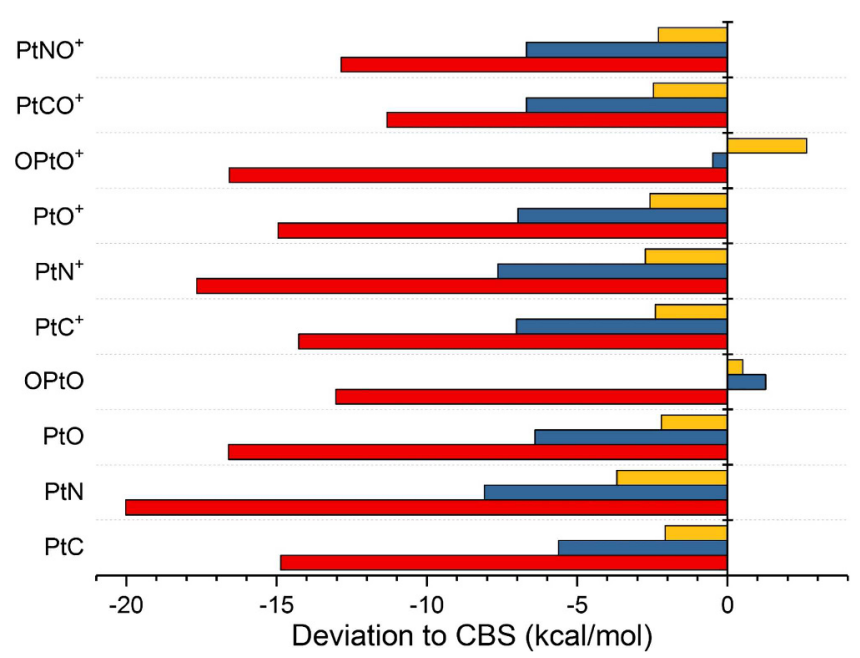

Fig. 3. Deviation of BDEs resulting from $\operatorname{CCSD}(\mathrm{T}) /$ def2-XVP with $X=S$ (red bars), TZ (blue bars), QZ (yellow bars) calculations from CBS-limit extrapolated using the def2-TZVP and def2-QZVP basis sets for all considered molecules.

uses $\alpha=7.88 . A$ is determined by solving the system of equations resulting from inserting $X=3$ and $X=4$ in Equation (4) for the use of triple zeta and quadruple zeta basis sets, respectively.

The correlation energy is extrapolated using

$$
E_{\mathrm{corr}}^{(\infty)}=\frac{X^{\beta} E_{\mathrm{corr}}^{(X)}-Y^{\beta} E_{\mathrm{corr}}^{(Y)}}{X^{\beta}-Y^{\beta}},
$$

with $\beta=3$. Summation of $E_{\mathrm{SCF}}^{(\infty)}$ and $E_{\mathrm{corr}}^{(\infty)}$ finally yields the total energy in the CBS-limit. In Figure 3 we depict the deviation of the BDEs from the CBS-limit extrapolated via the $\operatorname{CCSD}(\mathrm{T}) /$ def2-TZVP and $\operatorname{CCSD}(\mathrm{T}) /$ def2QZVP levels of theory for the various molecules in our test set for employing $\operatorname{CCSD}(\mathrm{T})$ in conjunction with the basis sets def2-SVP (cardinal number 2, red bars in Fig. 3), def2-TZVP (cardinal number 3, blue bars in Fig. 3) and def2-QZVP (cardinal number 4, yellow bars in Fig. 3). Note that all molecules were optimized at the TPSSh/def2-QZVP level of theory prior to computation of the BDEs. As expected, the deviation from the CBSlimit decreases with increasing basis set size. For almost all systems and basis set sizes the deviation is negative, hence the BDEs are underestimated. The exceptions are OPtO and its cation. For OPtO both $\operatorname{CCSD}(\mathrm{T}) / \operatorname{def} 2-$ TZVP and CCSD(T)/def2-QZVP levels of theory yield slightly overestimated BDEs. In case of its cation, the deviation is also positive for employing def2-QZVP and it is also larger than the absolute value of the deviation for employing the def2-TZVP basis set. Overall, the deviations from the CBS-limit range from 12.9 to $20 \mathrm{kcal} / \mathrm{mol}$, 1.3 to $8 \mathrm{kcal} / \mathrm{mol}$ and 0.5 to $3.69 \mathrm{kcal} / \mathrm{mol}$ for the def2SVP, def2-TZVP and def2-QZVP basis sets, respectively.

Multireference character of the molecular wave function. Whereas coupled cluster methods are known to capture contributions to the electronic energy from dynamic correlation quite accurately, systems with considerable static correlation might be described less
Table 2. $T 1, \% \mathrm{TAE}$ and $N_{\mathrm{FOD}}$ values for molecules in TS-BDE

\begin{tabular}{llll}
\hline Molecule & $T 1$ & \%TAE & $N_{\mathrm{FOD}}$ \\
\hline $\mathrm{PtO}$ & 0.048 & 13.01 & 0.86 \\
$\mathrm{PtO}^{+}$ & 0.059 & 14.0 & 0.61 \\
$\mathrm{PtN}^{+}$ & 0.074 & 17.8 & 1.43 \\
$\mathrm{PtN}^{+}$ & 0.077 & 20.0 & 1.42 \\
$\mathrm{PtC}$ & 0.037 & 11.5 & 0.46 \\
$\mathrm{PtC}^{+}$ & 0.04 & 10.89 & 0.42 \\
$\mathrm{PtNO}^{+}$ & 0.024 & 11.8 & 0.36 \\
$\mathrm{PtCO}^{+}$ & 0.022 & 4.11 & 1.30 \\
OPtO & 0.030 & 18.2 & 0.55 \\
OPtO $^{+}$ & 0.032 & 28.7 & 0.66 \\
\hline
\end{tabular}

well [57]. To this end, it is important to explore the system's multireference character in order to estimate the reliability of coupled cluster calculations with regard to the influence of static correlation. Several schemes have been proposed to do so, such as the T1 diagnostics [58], the \%TAE method [57] and the analysis of fractional orbital density (FOD) [59,60].

The \% TAE method uses the quantitative relationship between $\operatorname{CCSD}(\mathrm{T})$ and higher order coupled cluster theory and \% TAE is calculated by

$$
\% \mathrm{TAE}=100 \cdot\left(\frac{\operatorname{TAE}_{e}[\mathrm{CCSD}(T)]-\mathrm{TAE}_{e}[\mathrm{CCSD}]}{\operatorname{TAE}_{e}[\operatorname{CCSD}(T)]}\right)
$$

where $\operatorname{TAE}_{e}(M)$ is the total atomization energy of a molecule obtained with the method $M$.

Note that there are no definitive thresholds and also no guiding values particularly for the case of transition metals concerning acceptable values for $T 1$ and \%TAE. However, in general for systems consisting of atoms from the first two rows, values of $T 1>0.05$ and of \%TAE $>10$ are assumed to indicate that the system yields significant multireference character, affecting considerably the reliability of the $\operatorname{CCSD}(\mathrm{T})$ method.

The FOD method is a real-space measure of static electron correlation, in which fractional occupation numbers are determined via finite-temperature DFT also known as Fermi smearing method [60]. The fractional occupation numbers are used as weights in the aggregation of molecular orbitals in order to arrive at a modified electron density. Spatial integration of this weighted density yields a value $N_{\text {FOD }}$, which is used to quantify the influence, which omitting static correlation can have on the reliability of results [60].

Table 2 provides calculated T1, \% TAE and FOD values (based on TPSS/def2-SVP calculations) for all molecules considered in TS-BDE. We find that all \%TAE values are above the value of 10 , reaching values as high as 28.7 for $\mathrm{OPtO}^{+}$which would indicate a considerable multireference character of the systems. On the other hand, the $T 1$ value for $\mathrm{OPtO}^{+}$is 0.03 which is below 0.05 . Moreover, 7 out of $10 T 1$ values of the BDE test set are below 0.05 , which is in contrast to the \% TAE values.

Although somewhat controversial, these results suggest that static correlation may be of significant importance 
Table 3. Comparison of BDEs obtained with various variants of CCSD(T) calculations. Results obtained with CCSD(T)/def2QZVP are taken as reference values (leftmost column). For the other methods, we provide the relative differences with respect to these reference values (in units of $\mathrm{kcal} / \mathrm{mol}$ ). ${ }^{a}$ Using a ZORA Hamiltonian, see [63]; ${ }^{b}$ wave function.

\begin{tabular}{|c|c|c|c|c|c|}
\hline & \multicolumn{5}{|c|}{$\operatorname{CCSD}(\mathrm{T})$} \\
\hline Basis set: & def2-QZVP & $\mathrm{SARC}^{a}$ & $\mathrm{SARC}^{a}$ & def2-QZVP & def2-QZVP \\
\hline Use of ECP: & Yes & $\mathrm{No}$ & $\mathrm{No}$ & Yes & Yes \\
\hline Use of FC approximation: & Yes & No & Yes & No & Yes \\
\hline Reference $\mathrm{WF}^{b}$ : & $\mathrm{HF}$ & $\mathrm{HF}$ & $\mathrm{HF}$ & $\mathrm{HF}$ & PBE \\
\hline $\mathrm{PtC}$ & 0.00 & 0.19 & -0.32 & 0.22 & 0.78 \\
\hline $\mathrm{PtC}^{+}$ & 0.00 & -0.41 & -0.93 & 0.13 & 1.12 \\
\hline $\mathrm{PtO}$ & 0.00 & -3.25 & -3.65 & 0.13 & 0.56 \\
\hline $\mathrm{PtO}^{+}$ & 0.00 & -3.68 & -4.09 & 0.07 & -0.19 \\
\hline PtN & 0.00 & -2.39 & -2.87 & 0.11 & 2.49 \\
\hline $\mathrm{PtN}^{+}$ & 0.00 & -2.6 & -3.13 & 0.04 & 2.20 \\
\hline $\mathrm{PtCO}^{+}$ & 0.00 & -2.32 & -2.77 & 0.18 & 0.38 \\
\hline $\mathrm{PtNO}^{+}$ & 0.00 & -1.53 & -1.74 & -0.06 & 0.53 \\
\hline OPtO & 0.00 & 1.36 & 0.60 & 0.15 & 1.63 \\
\hline $\mathrm{OPtO}^{+}$ & 0.00 & -1.53 & -1.74 & -0.06 & 0.53 \\
\hline
\end{tabular}

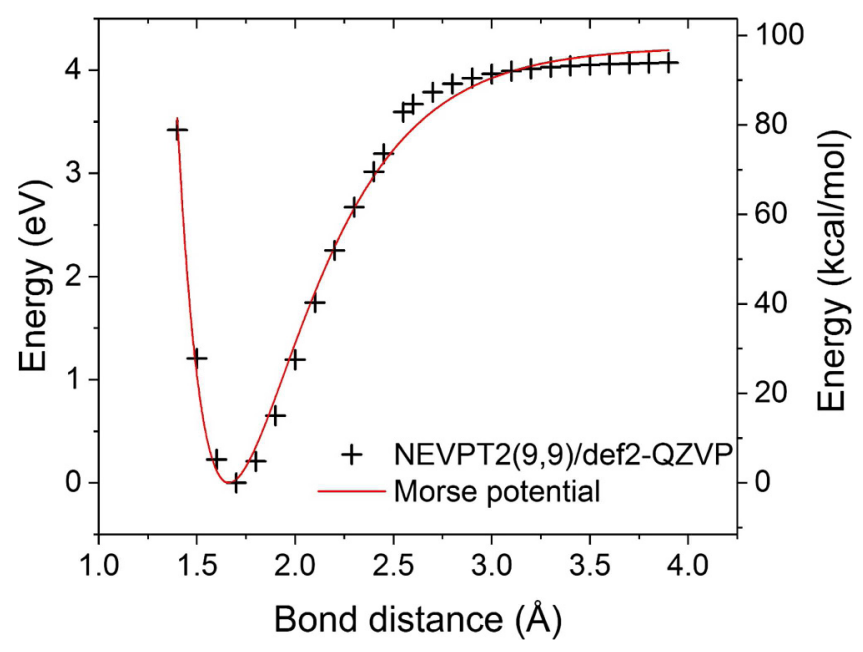

Fig. 4. Potential energy surface (PES) of PtN at the NEVPT2 $(9,9) /$ def2-QZVP level of theory (black crosses) and fitted Morse potential (red line).

for an accurate description of the considered molecules. This aspect definitely requires further investigation using multireference methods such as complete active space perturbation theory. In order to explore this issue at least tentatively, we picked PtN, i.e. the molecule yielding the highest FOD value (yielding also the second and fourth highest values with respect to $T 1$ and \% TAE, respectively), and performed a potential energy surface scan using the multi-reference approach NEVPT2. At the NEVPT2 $(9,9)$ /def2-QZVP level of theory and extrapolation to complete dissociation via fitting a Morse function to the calculated PES, we arrive at a BDE of $4.23 \mathrm{eV}(97.46 \mathrm{kcal} / \mathrm{mol})$, see also Figure 4 . The BDE calculated with CCSD(T) without ZPE and BSSE correction yields $3.75 \mathrm{eV}$ which is, however, closer to the experimental value of $3.84 \mathrm{eV}$ than the result obtained with NEVPT2.
Usage of the HF determinant as a reference wave function for post HF calculations. It has been suggested that the $\mathrm{HF}$ wave function might provide a bad reference for coupled cluster calculations [61], when it does not offer a qualitatively correct description of the electronic structure. Minenkov et al. investigated the effect of using different wave functions as reference for $\operatorname{CCSD}(\mathrm{T})$ calculations for transition metals in general [34]. Analogous to this work of Minenkov et al. [34] we employed the GGA density functional PBE $[45,62]$ to obtain a reference wave function used in a subsequent $\operatorname{CCSD}(\mathrm{T})$ calculation in order to explore the sensitivity of the considered BDEs on the reference wave function. The results are summarized in Table 3. The BDEs obtained at the CCSD(T)/def2-QZVP level of theory with a usual HF reference wave function are used as reference energies and only differences to these are given in the rightmost column of Table 3 for the case of a PBE reference wave function. Note that the $\operatorname{CCSD}(\mathrm{T}) /$ def2QZVP computations used as reference make use of both a frozen core (FC) approximation and effective core potentials (ECPs), see also the next section for a discussion on these approximations. In the case of using a PBE reference wave function the resulting BDEs (almost) increase for all considered molecules. The only exception is $\mathrm{PtO}^{+}$for which the BDE slightly decreases by $0.19 \mathrm{kcal} / \mathrm{mol}$. For the other molecules the increases in BDE due to change of the reference wave function range from 1.16 to $2.49 \mathrm{kcal} / \mathrm{mol}$ yielding a considerable influence of the choice of reference wave function. However, the MAD with respect to the experimental values is $9.49 \mathrm{kcal} / \mathrm{mol}$ yielding a slight increase of about $0.5 \mathrm{kcal} / \mathrm{mol}$ upon employing a PBE reference wave function.

Treatment of core electrons. $\mathrm{CCSD}(\mathrm{T})$ calculations are typically performed using a FC approximation, especially for larger molecular species. That means that inner shell electrons (46 in the case of $\mathrm{Pt}$ ) are not included in the calculation of the correlation energy, which saves computational time but, obviously, represents an approximation. Moreover, in the case of the def2-QZVP basis set, 60 of 78 
electrons of $\mathrm{Pt}$ are modelled using an ECP and are thus not treated explicitly in the SCF computation.

In order to assess the errors introduced by these approximations, we replaced the def2-QZVP basis set by the all-electron basis set SARC proposed by Neese et al. [64] which was especially developed for transition metals. Since the employed ECP also captured relativistic effects to some extent, they were taken into account by using a zeroth-order regular approximation (ZORA) Hamiltonian. In Table 3 we compare BDEs resulting from $\operatorname{CCSD}(\mathrm{T})$ calculations with (a) both a FC approximation and employing an ECP by using the def2-QZVP basis set, (b) no FC approximation and using the all-electron SARC basis set, (c) a FC approximation but using the all-electron SARC basis set and (d) no FC approximation but using an ECP. The results in case (a) were used as reference and only the deviations to this reference energy are given for the other cases in Table 3 for all considered molecules. Whereas explicitly including correlation of core electrons in case (d) leads in most cases to slightly larger BDEs ranging from 0.07 to $0.22 \mathrm{kcal} / \mathrm{mol}$, the resulting deviations can lead to both larger and smaller BDEs in the case of using the all-electron basis set SARC instead of def2QZVP, see the results for cases (b) and (c) in Table 3. In cases (b) and (c) the deviations are also considerably smaller regarding their absolute values ranging from 0.19 up to $4.09 \mathrm{kcal} / \mathrm{mol}$. Exceptions are $\mathrm{OPtO}$ and $\mathrm{PtC}$ with slightly increased BDEs.

\section{Concluding remarks}

We systematically compared the accuracy of various electronic structure methods regarding optimized bond lengths and resulting bond dissociation energies (BDEs) of small platinum-containing molecules in the gas phase. The resulting accuracies were assessed using experimental data available for these molecules. We found that the meta hybrid density functionals TPSSh and tHCTH are the most accurate methods concerning bond lengths yielding MADs of 0.3 and $0.45 \mathrm{pm}$, respectively. In comparison, $\operatorname{CCSD}(\mathrm{T})$ results in a MAD of $0.62 \mathrm{pm}$. Apart from $\operatorname{CCSD}(\mathrm{T})$, ab initio methods and also double hybrid functionals yield considerably larger deviations from experimental values than these density functionals. The most accurate GGA functionals TPSSPBE, TPSSPW91, TPSSTPSS and TPSSVP86 with MADs of about $0.8 \mathrm{pm}$ may still be a suitable choice for large-scale computations including geometrical (pre-)screening procedures. Furthermore, we found that also the precision of the considered methods goes - in most cases - hand in hand with their accuracy. In particular, it turned out that the most accurate methods are also the most precise ones.

Concerning the BDEs, we note that all considered methods differ considerably from experimental data. The DFT method with the smallest MAD of $6.97 \mathrm{kcal} / \mathrm{mol}$ is the combination of the GGA functional TPSS and the LDA functional VWN5 for computing exchange and correlation energy, respectively. Also the precisions are of the same order of magnitude as the accuracies. Hence, if - as usually - both accuracy and precision are wanted, our results interestingly suggest the use of functionals at the GGA level and/or even lower for the computation of BDEs in contrast to both computationally considerably more demanding and methodologically more involved methods. Especially $\operatorname{CCSD}(\mathrm{T})$ resulted in a mean average deviation of $8.87 \mathrm{kcal} / \mathrm{mol}$ concerning the assessed BDEs. Regarding the latter, we discussed various possible sources of error which could underlie this high value in contrast to the high accuracy typically delivered by coupled-cluster methods for molecules consisting of first and second row elements. In particular, we find that

1. the considered systems may exhibit notable static correlation which may considerably affect also the resulting bond dissociation energies - an issue which requires further exploration in future work,

2. contributions to individual deviations from experimental reference values of the considered energies due to (a) limited basis set size, (b) the use of a HF reference wave function and (c) correlation of core electrons each may yield a few $\mathrm{kcal} / \mathrm{mol}$,

3. but appear nevertheless too small to account for the entire difference between experimental and $\operatorname{CCSD}(\mathrm{T})$ results on the considered BDEs, even if taken together.

However, it must not be forgotten that experimentally determined quantities cannot be regarded exact either and have been criticized earlier, particularly concerning transition metals [11]. Delivering reliable benchmark data for transition metal compounds hence appears to remain a difficult task for both experiment and theory, and platinum-containing molecules are definitely no exception. Although hardly a relief, our results at least illustrate and put emphasis on the fact that $\operatorname{CCSD}(\mathrm{T})$ cannot per se be regarded as a gold standard for the case of platinumcontaining molecules; at least not with the same confidence as it may be admissible for molecules consisting of main group elements.

Open access funding provided by Austrian Science Fund (FWF). This work was supported by the Austrian Ministry of Science BMWF as part of the University infrastructure program of the scientific computing platform LFU Innsbruck and by the Austrian Fund Agency (FWF P 30355). Moreover, we would like to express our gratitude to Prof. Michael Probst for his continuous guidance and patient support on various occasions.

Open Access This is an open access article distributed under the terms of the Creative Commons Attribution License (http://creativecommons.org/licenses/by/4.0/), which permits unrestricted use, distribution, and reproduction in any medium, provided the original work is properly cited.

\section{Author contribution statement}

All authors contributed equally to the paper. 


\section{References}

1. D. Wang, S.J. Lippard, Nat. Rev. Drug Discovery 4, 307 (2005)

2. H.P. Varbanov, M.A. Jakupec, A. Roller, F. Jensen, M. Galanski, B.K. Keppler, J. Med. Chem. 56, 330 (2013)

3. S. Medici, M. Peana, V.M. Nurchi, J.I. Lachowicz, G. Crisponi, M.A. Zoroddu, Coord. Chem. Rev. 284, 329 (2015)

4. L. Kelland, Nat. Rev. Cancer 7, 573 (2007)

5. P. Sarmah, R.C. Deka, Int. J. Quantum Chem. 108, 1400 (2008)

6. G. Markus, A.J. Michael, K.K. Bernhard, Curr. Med. Chem. 12, 2075 (2005)

7. R.J. Bartlett, M. Musiał, Rev. Mod. Phys. 79, 291 (2007)

8. G.D. Purvis III, R.J. Bartlett, J. Chem. Phys. 76, 1910 (1982)

9. J.A. Pople, M. Head-Gordon, K. Raghavachari, J. Chem. Phys. 87, 5968 (1987)

10. X. Xu, W. Zhang, M. Tang, D.G. Truhlar, J. Chem. Theory Comput. 11, 2036 (2015)

11. Z. Fang, M. Vasiliu, K.A. Peterson, D.A. Dixon, J. Chem. Theory Comput. 13, 1057 (2017)

12. I. Georgieva, N. Trendafilova, N. Dodoff, D. Kovacheva, Spectrochim Acta Part A 176, 58 (2017)

13. M. Kokoschka, J. Galgonek, J. Vondrasek, P. Hobza, Phys. Chem. Chem. Phys. 18, 4051 (2016)

14. W. Liu, R. Franke, J. Comput. Chem. 23, 564 (2002)

15. M. Citir, R.B. Metz, L. Belau, M. Ahmed, J. Phys. Chem. A 112, 9584 (2008)

16. T. Okabayashi, T. Kurahara, E.Y. Okabayashi, M. Tanimoto, J. Chem. Phys. 136, 174311 (2012)

17. X.-G. Zhang, P.B. Armentrout, J. Phys. Chem. A 107, $8904(2003)$

18. X.-G. Zhang, P.B. Armentrout, Eur. J. Mass Spectrom. 10, $963(2004)$

19. M.J. Frisch, M. Head-Gordon, J.A. Pople, Chem. Phys. Lett. 166, 275 (1990)

20. M.J. Frisch, M. Head-Gordon, J.A. Pople, Chem. Phys. Lett. 166, 281 (1990)

21. M. Head-Gordon, J.A. Pople, M.J. Frisch, Chem. Phys. Lett. 153, 503 (1988)

22. S. Sæbø, J. Almlöf, Chem. Phys. Lett. 154, 83 (1989)

23. M. Head-Gordon, T. Head-Gordon, Chem. Phys. Lett. 220, 122 (1994)

24. J.A. Pople, J.S. Binkley, R. Seeger, Int. J. Quantum Chem. 10, 1 (1976)

25. J.A. Pople, R. Seeger, R. Krishnan, Int. J. Quantum Chem. 12, 149 (1977)

26. R. Krishnan, J.A. Pople, Int. J. Quantum Chem. 14, 91 (1978)

27. R. Krishnan, M.J. Frisch, J.A. Pople, J. Chem. Phys. 72, 4244 (1980)

28. J.A. Pople, R. Krishnan, H.B. Schlegel, J.S. Binkley, Int. J. Quantum Chem. 14, 545 (1978)

29. R.J. Bartlett, G.D. Purvis, Int. J. Quantum Chem. 14, 561 (1978)

30. G.E. Scuseria, C.L. Janssen, H.F. Schaefer III, J. Chem. Phys. 89, 7382 (1988)

31. G.E. Scuseria, H.F. Schaefer III, J. Chem. Phys. 90, 3700 (1989)

32. J.A. Pople, M. Head-Gordon, K. Raghavachari, J. Chem. Phys. 87, 5968 (1987)
33. F. Weigend, R. Ahlrichs, Phys. Chem. Chem. Phys. 7, 3297 (2005)

34. Y. Minenkov, E. Chermak, L. Cavallo, J. Chem. Theory Comput. 12, 1542 (2016)

35. V.N. Staroverov, G.E. Scuseria, J. Tao, J.P. Perdew, J. Chem. Phys. 119, 12129 (2003)

36. A.D. Boese, N.C. Handy, J. Chem. Phys. 116, 9559 (2002)

37. J. Heyd, G.E. Scuseria, J. Chem. Phys. 121, 1187 (2004)

38. J. Heyd, G.E. Scuseria, J. Chem. Phys. 120, 7274 (2004)

39. J. Heyd, J.E. Peralta, G.E. Scuseria, R.L. Martin, J. Chem. Phys. 123, 174101 (2005)

40. T.M. Henderson, A.F. Izmaylov, G. Scalmani, G.E. Scuseria, J. Chem. Phys. 131, 044108 (2009)

41. A.F. Izmaylov, G.E. Scuseria, M.J. Frisch, J. Chem. Phys. 125, 104103 (2006)

42. A.V. Krukau, O.A. Vydrov, A.F. Izmaylov, G.E. Scuseria, J. Chem. Phys. 125, 224106 (2006)

43. A.D. Becke, J. Chem. Phys. 98, 5648 (1993)

44. J. Tao, J.P. Perdew, V.N. Staroverov, G.E. Scuseria, Phys. Rev. Lett. 91, 146401 (2003)

45. J.P. Perdew, K. Burke, M. Ernzerhof, Phys. Rev. Lett. 77, 3865 (1996)

46. J.P. Perdew, J.A. Chevary, S.H. Vosko, K.A. Jackson, M.R. Pederson, D.J. Singh, C. Fiolhais, Phys. Rev. B 46, 6671 (1992)

47. J.P. Perdew, K. Burke, Y. Wang, Phys. Rev. B. 54, 16533 (1996)

48. S.H. Vosko, L. Wilk, M. Nusair, Can. J. Phys. 58, 1200 (1980)

49. J.P. Perdew, Phys. Rev. B 33, 8822 (1986)

50. S. Grimme, J. Chem. Phys. 124, 16 (2006)

51. G. Frenking, I. Antes, M. Böhme, S. Dapprich, A.W. Ehlers, V. Jonas, A. Neuhaus, M. Otto, R. Stegmann, A. Veldkamp, S.F. Vyboishchikov, Rev. Comput. Chem. 8, 63 (1996)

52. F.A. Hamprecht, A.J. Cohen, D.J. Tozer, N.C. Handy, J. Chem. Phys. 109, 6264 (1998)

53. H.L. Schmider, A.D. Becke, J. Chem. Phys. 108, 9624 (1998)

54. C. Riplinger, B. Sandhoefer, A. Hansen, F. Neese, J. Chem. Phys. 139, 134101 (2013)

55. K.L. Bak, P. Jørgensen, J. Olsen, T. Helgaker, W. Klopper, J. Chem. Phys. 112, 9229 (2000)

56. F. Neese, Wiley Interdiscip. Rev.: Comput. Mol. Sci. 2, 73 (2012)

57. W. Jiang, N.J. DeYonker, A.K. Wilson, J. Chem. Theory Comput. 8, 460 (2012)

58. T.J. Lee, P.R. Taylor, Int. J. Quantum Chem. 36, 199 (1989)

59. C.A. Bauer, A. Hansen, S. Grimme, Chem. Eur. J. 23, 6150 (2017)

60. S. Grimme, A. Hansen, Angew. Chem. Int. Ed. 54, 12308 (2015)

61. F. Neese, D.G. Liakos, S. Ye, J. Biol. Inorg. Chem. 16, 821 (2011)

62. J.P. Perdew, K. Burke, M. Ernzerhof, Phys. Rev. Lett. 77, 3865 (1996) [Erratum: Phys. Rev. Lett. Vol. 78, p. 1396, 1997]

63. E.V. Lenthe, J.G. Snijders, E.J. Baerends, J. Chem. Phys. 105, 6505 (1996)

64. D.A. Pantazis, X.-Y. Chen, C.R. Landis, F. Neese, J. Chem. Theory Comput. 4, 908 (2008) 